\title{
A Study on the Algorithm of Supply Chain Protection Based on Zero-sequence Compensation
}

\author{
JiangJun Yuan ${ }^{1}$ \\ 1.Department of Shangmao, Zhejiang Technical Institute of Economics, Hangzhou, \\ Zhejiang, 310018, China \\ welleb@qq.com
}

\begin{abstract}
To deal with the faulted line selection in the single-phase to ground faults caused by neutral non-effective grounding in the distribution network, a new method by using zero-sequence admittance is proposed in this paper. Compensation admittance, which amplifies the difference in zero-sequence admittance between the faulted line and the unfaulted line, is convenient for the formation of the criteria for value setting in engineering. To eliminate the blind zone, combining the criterion of the susceptance error in the compensated admittance with the phase angle criterion helps form a new algorithm to identify the single-phase to ground faults in the neutral non-effectively grounded system. Results of simulation tests show that the algorithm has nothing to do with the network structure and parameters, fault conditions and fault resistance, significantly escalating the successful selection of the faulted line.
\end{abstract}

Keywords: zero-sequence compensation; power supply line; line fault

\section{Introduction}

Automatic detection and the removal of the faulted line are important to the automatic distribution of power and the reliability of power supply. To identify the faulted line is an important issue because, in the neutral non-effectively grounded distribution system, the ground fault current is actually so small that it is not a must to remove the faulted line instantly. In this case, the important current load can be shifted onto other power supply through restructuring the distribution network before the faulted line is tripped. The faulted line - caused by reasons of the system, such as the current load that is unable to be shifted due to the power line topology, the positions of switches and capacity margin of adjacent lines - can continue its operation for a period. In this case, the time of power failure on the loads can be shortened. Therefore, the reliability of power supply is ensured.

Many methods have been raised and applied to the line selection in the neutral non-effectively grounded system. Of the available faulted line selection methods, the one based on stationary signals [1-3] is widely applied, typically including the zero-sequence 
admittance method [4], current injection method [5], zero-sequence active component method [6] and the quintuple harmonic method [7].

In the zero-sequence admittance method, the phases of zero-sequence currents are compared. The current phase of the faulted line is 180 o different from that of the unfaulted line. This method is applicable when the neutral point in the system is not grounded. However, when the neutral point is grounded by the arc suppression coil and the compensation degree is relatively higher, zero-sequence currents of all lines will be approximately the same [8-9].

The purpose of this paper is to design a method of single-phase ground protection with high sensitivity based on the stationary signal, realizable on the local fixed feeder terminal unit. To achieve this goal, the method based on zero-sequence admittance is considered to be a good choice. However, because of the complicated factors at the site, the differentiating margins between the unfaulted line and the faulted line should be increased in these existing methods that are based on admittance measurement. In this paper, based on the discussion on the ideas of line selection by zero sequence admittance, a new method by zero-sequence compensated admittance is proposed. Theoretical analyses and simulation results show that it is a sensitive and reliable single-phase ground protection method in the neutral non-effectively grounded system.

\section{The Principle for the Algorithm of Zero-sequence Admittance Line Selection}

In the typical distribution network system as shown in Figure 1, it is assumed that the single-phase ground fault occurs on Line 1.

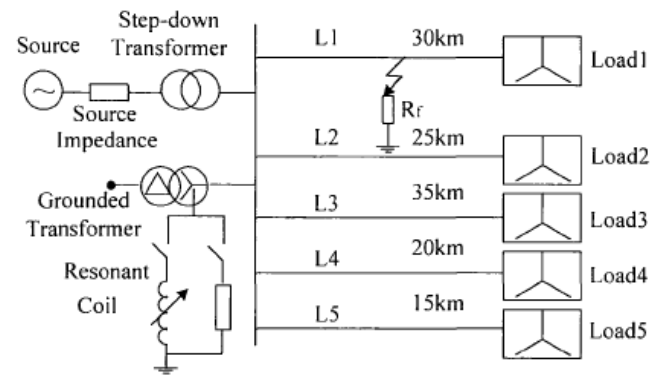

Figure 1. Neutral Non-effectively Grounded System

The measured zero-sequence admittance of the unfaulted line $i \quad(i \neq 1)$ equals its zero-sequence admittance, which includes the susceptance of natural capacitance and leakage conductance. In simplified representation, the subscript ' 0 ' of the zero-sequence susceptance and the conductance parameters are ignored, as shown in Equation (1) below: 


$$
\begin{aligned}
& Y_{\text {comp }}=Y_{\text {meas }}-Y_{\text {set }}=-\left(G_{s}+\sum_{i=2}^{n} G_{i}\right)-j v \sum_{i=1}^{n} B_{i} \\
& =G_{i}+j B-j B_{i} \text { ocuc } \\
& =G_{i}
\end{aligned}
$$

The damping ratio of the overhead line, $\mathrm{d}$, which is the ratio between the effective current components of the residual currents and the total capacitance current of the network, is roughly in the range from $3 \%$ to $10 \%$; while the damping ratio of the electric cable is roughly in the range of $2 \%$ to $4 \%$, or probably up to $10 \%$ when the line insulation has become aged. Therefore, the range of admittance angle goes generally between $0 \mathrm{o}$ and $90 \mathrm{o}$. For this reason, the admittance of the unfaulted line is always in Quadrant I on the admittance plane.

As for the faulted line, when it is assumed that zero-sequence conductance of the arc suppression coil is GS, and its electrical susceptance is BS, the zero-sequence measured admittance is shown as Equation (2) below:

$$
Y_{\text {maes } 1}=-\frac{I_{i}}{U_{i}}=-\left(G+\sum_{i=2}^{n} G_{i}\right)-j\left(G_{s}+\sum_{i=2}^{n} B_{i}\right)
$$

The value of the measured zero-sequence admittance of the faulted line equals the sum, but opposite in sign, of the admittance of the suppression coil and that of the unfaulted line.

Assuming $B=-(1-v) \sum_{i=1}^{n} B_{i}$, where $v$ is detuning degree, we will lead to:

$$
Y_{\text {maes } 1}=-\frac{I_{i}}{U_{i}}=-\left(G_{s}+\sum_{i=2}^{n} G_{i}\right)-j\left[v \sum_{i=2}^{n} B_{i}+(v-1) B_{1}\right]
$$

Detuning degree $v$ is the ratio of the reactive current component in the residual current and the total capacitance current in the network. The sign and value of $v$ indicate the different ways of operation and the degree of deviating from the resonant state in the resonant equivalent circuit. When the system is fully compensated, $v$ is equal to zero; when the system is in overcompensation, $v$ is less than zero; and when the system is in under-compensation, $v$ is greater than zero. When the neutral point in the system is not grounded, $v$ is equal to 1 .

Whatever the compensation mode is taken, the conductance of the zero sequence admittance can be expressed as: $G_{1}=-\left(G_{x}+\sum_{i=2}^{n} G_{i}\right)<0$. Namely, the vector of the zero sequence admittance is in the left part of the admittance plane. For the neutral non-grounded system $B_{s}=G_{s}=0$, the admittance vector is still in the left part of the plane.

Therefore, the zero sequence admittance can theoretically be utilized to accurately identify the faulted line. However, the admittance angle in the line is primarily affected by the conductance. When $G$ is very small, the admittance angle of the unfaulted line is close to $90^{\circ}$. Under the condition of full compensation, the susceptance of zero sequence admittance in the faulted line equals its natural susceptance; and the conductance is equal to the sum total conductance of the remaining line and the conductance of the arc suppression coil, and the sum is negative. In generally, the conductance is always far less than the susceptance. In this case, the measured zero-sequence admittance may constantly be in Quadrant II in the admittance plane, and close to the positive imaginary axis; therefore admittance angle is close to $90^{\circ}$. From the above analysis, it can be known that the zone close to the imaginary 
axis in the complex plane is prone to misjudgments. Usually, the arc suppression coil grounded network is actually running certain over-compensation. Concerning the unfaulted line, measured admittance is generally positioned near the imaginary axis. Since the conductance, compared with the susceptance, is smaller and uncertain, it is difficult to set the criterion angle that differentiating faulted line from the unfaulted line. Consequently, the sensitivity of this criterion desires improvement.

\section{The Grounded Protection with Zero-sequence Compensated Admittance}

\subsection{Protection Principle}

To avoid the overlap of the zone of the zero-sequence admittance angle of the faulted line and that of the unfaulted line, one solution is to adjust the setting of the action zone and that of the non-action zone in the complex plane to avail certain margins. If this approach is adopted, the uncertainty zone would be expanded and the selective accuracy can hardly be improved. To solve the problem thoroughly, other approaches should be taken.

In spite of the nondeterminacy existing in the line conductance, zero-sequence susceptance of any line can be calculated according to the design parameters or to on-site measurements. In fact, $B_{\text {set }}=b_{0} L=\omega C_{0} L$, where $b_{0}$ represents the zero-sequence susceptance of per unit length (unit: s/km) of the line, $L$ represents the length of the line (unit: $\mathrm{km}$ ). If the reference susceptance is set as $Y_{\text {set }}=j B_{\text {set }}$, the compensated admittance can be defined as:

$$
Y_{\text {comp }}=Y_{\text {comp }}-Y_{\text {set }}
$$

When the single-phase ground fault occurs, the compensation admittance of the unfaulted line is:

$$
Y_{\text {comp }}=Y_{\text {meas }}-Y_{\text {set }}=G_{i}+j B_{i}-j B_{i}=G_{i}
$$

That is, with only the positive conductance part left, the compensation admittance angle equals $0^{\circ}$

For the faulted line, the compensation admittance is :

$$
Y_{\text {comp }}=Y_{\text {meas }}-Y_{\text {set }}=-\left(G_{s}+\sum_{i=2}^{n} G_{i}\right)-j v \sum_{i=1}^{n} B_{i}
$$

When $v=0$ (fully compensated), $\left.v n \sum_{i=1} B_{i}\right)>0$. Clearly, with only the negative part of the conductance left, the compensation admittance angle equals $180^{\circ}$.

When $v<0$ (over-compensated), the conductance part of $Y_{\text {comp }}$ is $-\left(G_{s}+\sum_{i=2}^{n} G_{i}\right)<0$, the susceptance part of $Y_{\text {comp }}$ is $\left.v \sum_{i=1} B_{i}\right)<0$, the compensation admittance angle is between $90^{\circ}-180^{\circ}$ 
When $v>0$ (under-compensated), the conductance part of $Y_{\text {comp }},-\left(G_{s}+\sum_{i=2}^{n} G_{i}\right)<0$,

the susceptance part $\left.v \sum_{i=1} B_{i}\right)<0$, The compensation admittance angle is between $180^{\circ}-270^{\circ}$ 。

From the above analysis, it can be seen that, when there is nearly no loss on the unfaulted line, the phase angle of the compensation admittance is close to the positive imaginary axis or the negative imaginary axis. When the system operates away from the resonance point, the phase angle of the compensation admittance is close to the compensation angle of the faulted line. Therefore, it is possible to appropriately narrow the zone for fault identification. The problem that follows is, when the system is operating away from the resonance point, the compensation admittance angle of the faulted line may be outside the action zone.

But it is observed that, under the conditions mentioned above, for the faulted line, the susceptance of its compensated admittance is comparable with its zero-sequence admittance. But for the unfaulted line, the compensated admittance is zero theoretically. Therefore, the faulted line can be directly identified by the ratio of the susceptance of the compensation admittance to the susceptance of the faulted line as long as the measurement error is within the allowable range. In this way, the adduction angle of the fault identification zone can be set according to the margin of the measurement error. Yet there is still another problem to be solved. Due to accurate compensation, small is the amplitude of the compensated admittance of the unfaulted line that approximates to no power loss. In this case, the admittance angle can be any value due to the quantization error, and may enter the action zone. In fact, there always exist active power losses of the arc suppression coil, so the compensation admittance of the faulted line can not be zero. In other words, there is always a positive minimum value for the compensation admittance of the faulted line. If an action circle is generated with this minimum value as the radius in the admittance plane, the compensation admittance of the faulted line will never enter the action circle. Based on the above analysis, the action plane of compensation admittance is shown in Figure 2.

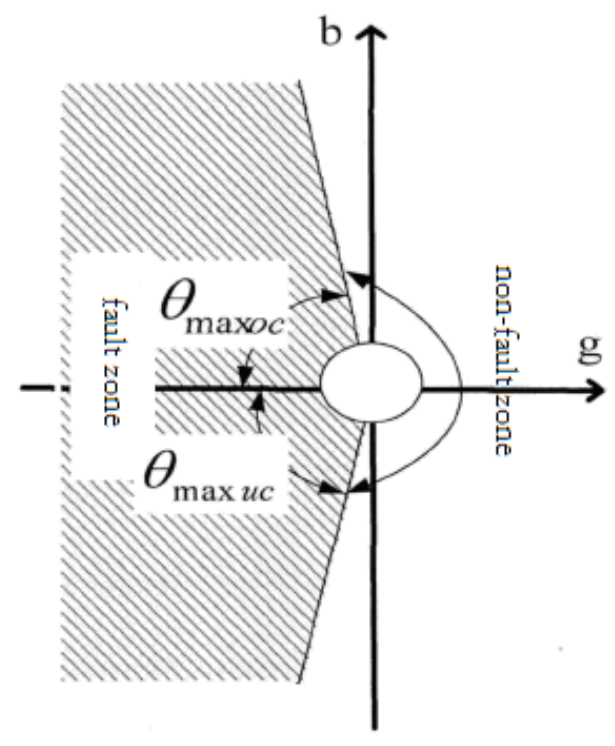

Figure 2. The Action Zone 
In Figure 2, the subscript of the action angle "oc" refers to over compensation; the subscript " $u c$ " means under compensation.

When the ratio of compensation susceptance to the set susceptance exceeds a certain threshold value, the faulted line can be directly confirmed. Otherwise, it should be judged according to the action zone in Figure 2. When the admittance angle is in the shaded area in the left half of the plane, the line is judged as the faulted line; otherwise, it is judged as the unfaulted line.

Before the confirmation of the faulted line according to Figure 2, the compensation admittance should first be normalized. The zero-sequence susceptance should be chosen as reference value, for example:

$$
Y_{p e r}=\frac{Y_{\text {comp }}}{B_{\text {set }}}
$$

In Formula (7), the subscript "per" refers to per unit length, the subscript "comp" to the compensation. If the compensation admittance after normalization is in the shaded area, the faulted line is confirmed. If the compensation admittance of all lines falls into the non-action zone, and does not reset after a period when the zero-sequence voltage is lower than the threshold value, the busbar fault is confirmed.

\subsection{Protection Setting}

First, the radius of the small circle in Fig 2 is to be set.

According to Formula (6), when all the unfaulted lines are without power loss and the arc suppression coil are operating under the resonance state, the amplitude of the compensation admittance of the faulted line drops to the minimum, equaling the conductance component $G_{s}$ of the arc suppression coil. Usually, the arc suppression coil has some active loss, which covers $1.5 \%-2.0 \%$ of the compensation capacitor. For this reason, $\mathrm{G}_{\mathrm{S}}=1.5-2.0 \% \times(1-\mathrm{v}) \mathrm{B}_{\Sigma}$. Therefore,

$$
Y_{p e r}=\frac{G_{s}}{B_{1}}=\frac{1.5 \sim 2.0 \times(1-v) B_{\Sigma}}{B_{1}}
$$

From Formula (8), to make the minimum of $Y_{p e r}$ r, the line must be without power loss and $B_{1}=B_{\Sigma}$. That is to say, the susceptance of the faulted line almost takes up the most part of the total susceptance in the system. Under the resonance condition, there is $v=0$, thus $\left|Y_{p e r}\right|_{\text {min }}=1.5 \sim 2.0 \%$. Actually, the two conditions mentioned above can hardly be satisfied at the same time. Therefore, when the radius of the small circle is set as 0.015 , any compensated admittance of the faulted line will not fall in the circle.

Next is to set the boundaries of the action zone. Suppose the measurement error of the susceptance is no more than 20\%; and under this situation, if the ratio of the compensation susceptance to the line susceptance is above $20 \%$, the line can be judged as the faulted line. Contrastively, for the unfaulted line, if the ratio is less than $20 \%$ and the effect of the line conductance cannot be ignored, the admittance angle will be in Quadrant I or Quadrant IV on the complex plane. Based on the above analysis, in the extreme case that the line is without power loss and the susceptance error is above 1.5, the admittance angle will be on the positive imaginary axis or the negative imaginary axis on the complex plane in accordance with the signs of the error.

For the faulted line, when $|v|$ is above $20 \%, B_{\text {comp }}>20 \% B_{\Sigma}$. At this time, even in the most unfavorable case, that is, $B_{1}=B_{\Sigma}$, the faulted line can still be identified by the 
susceptance error. Only when $|v|$ is less than $20 \%$, alternative measures are to be taken to locate the faulted line. Obviously, when $|v|$ is close to zero, that is, when the system is operating in the resonance state, the compensation admittance angle is close to $180^{\circ}$, which is the most sensitive action angle. When $|v|$ becomes larger, the compensation admittance angle will be more and more approaching the positive imaginary axis or the negative imaginary axis, which is the zone of unfaulted line. Therefore, only the maximal value of $|v|$ needs to be examined to obtain the corresponding compensation admittance angle. In such a way the boundary of the action zone is set.

According to Formula (6) and Formula (7),

$$
\theta_{\max o c}=\arctan \frac{0.2}{1.5 \% \times(1+0.2)}=84.86^{\circ}
$$

The angle between $Y_{p e r}$ and the negative real axis is:

$$
\theta_{\max }=\arctan \frac{|v| B_{\Sigma}}{G_{s}+\sum_{i=2}^{n} G_{i}}
$$

Obviously, to some extent of compensation, when there is no loss on the unfaulted line and $G_{s}$ is the minimum value, the angle will be maximal. In other words, the angle to the imaginary axis will be minimal, just as what discussed above. Therefore,

$$
\theta_{\max }=\arctan \frac{|v|}{1.5 \% \times(1-v)}
$$

When $|v|$ reaches the maximal value, namely $20 \%$, Formula (10) will obtain the maximal value in the possible value range. As to overcompensation, there is

$$
\theta_{\max o c}=\arctan \frac{0.2}{1.5 \% \times(1+0.2)}=84.86^{\circ}
$$

Similarly, under undercompensation the action boundary angle can be set as:

$$
\theta_{\max o c}=\arctan \frac{0.2}{1.5 \% \times(1-2)}=86.57
$$




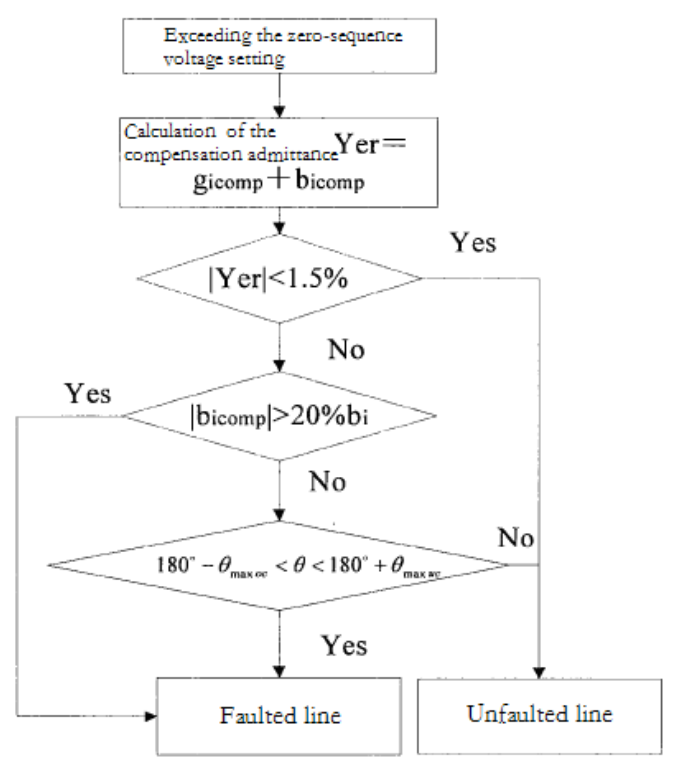

\section{Figure 3. Flowchart of the Single-phase Ground Protection based on Zero-sequence Compensation Admittance}

Now, all the action boundaries are defined. The basic process of protection is shown in Figure 3.

\subsection{Simulation Study}

To verify the algorithm proposed in this paper, EMTP is used to establish a system model as shown in Fig 1, which contains five lines from L1 to L5. The parameters for the system model are set as follows: power supply: $110 \mathrm{kV}$; transformer: $\mathrm{Y} / \Delta$ connection, $110 \mathrm{Kv} / 10.5 \mathrm{kV}$; distribution transformer parameters: $10 \mathrm{kV} / 6.3 \mathrm{kV}$; line: zero-sequence distributed capacitance $C_{0}=0.006 \mathrm{uF} / \mathrm{km}$; zero-sequence reactance on the line $X_{0}=0.982 \Omega / \mathrm{km}$; loads: $\mathrm{Z}_{\mathrm{Ld}}=240+\mathrm{j} 180 \Omega$. The length of each line is shown in Fig 1. Sample frequency is $4.8 \mathrm{kHz}$. This relatively high sample frequency can extract high frequency transient current to compare the proposed method in this paper with the method based on transient current.

By changing the system parameter setting, the neutral non-grounded system, the arc suppression coil grounded system and the high resistance grounded system can be simulated. Of the three systems, the focus is on the arc suppression coil grounded system:

1) Changing the inductance of arc suppression coil to change the detuning degree so as to simulate the condition of resonance compensation, overcompensation and under-compensation. The active loss of the arc suppression coil is set as $1.5 \%$.

2) Adjusting the line parameters, including the line length and active loss to simulate the different situations of the practical system (such as the uneven line length, and the overhead line polluted by varying degrees.)

3) Changing the grounding resistance to test the capacity to endure transition impedance of the algorithm. 
Table 1. Statistics of Typical Line Fault Identification

\begin{tabular}{|c|c|c|c|c|c|c|}
\hline $\begin{array}{l}\text { Neutral grounded } \\
\text { mode }\end{array}$ & $\begin{array}{l}\text { Damping } \\
\text { rate }(\%)\end{array}$ & $\begin{array}{l}\text { Ratio of faulted } \\
\text { line length to the } \\
\text { whole network } \\
\text { line }(\%)\end{array}$ & $\begin{array}{l}\text { ground } \\
\text { resistance } \\
(\Omega)\end{array}$ & $\begin{array}{l}\text { bl } \\
\text { comp/bl } \\
(\%)\end{array}$ & $\begin{array}{l}\text { compensation } \\
\text { admittance angle } \\
\left({ }^{\circ}\right)\end{array}$ & $\begin{array}{l}\text { Identification } \\
\text { results }\end{array}$ \\
\hline Non-grounded & 2.6 & 30 & $1 \mathrm{k}$ & 229 & Excluded & Line fault \\
\hline $\begin{array}{l}\text { Overcompensation } \\
\mathrm{v}=-3 \%\end{array}$ & 2.6 & 20 & 5 & 17 & 127.2 & Line fault \\
\hline $\begin{array}{l}\text { Overcompensation } \\
v=-4 \%\end{array}$ & 0 & 90 & 100 & 4.2 & 111.3 & Line fault \\
\hline $\begin{array}{l}\text { Overcompensation } \\
\mathrm{v}=-4 \%\end{array}$ & 7 & 80 & $100 \mathrm{k}$ & 5.3 & 118.5 & Line fault \\
\hline $\begin{array}{l}\text { Overcompensation } \\
\mathrm{v}=-4 \%\end{array}$ & 2.6 & 80 & 5 & 5.2 & 113.2 & Line fault \\
\hline $\begin{array}{l}\text { Overcompensation } \\
\mathrm{v}=-10 \%\end{array}$ & 2.6 & 5 & 5 & 202 & Excluding & Line fault \\
\hline $\begin{array}{l}\text { Overcompensation } \\
\mathrm{v}=-30 \%\end{array}$ & 1 & 60 & 5 & 52 & Excluding & Line fault \\
\hline $\begin{array}{l}\text { resonance } \\
\text { compensation } \mathrm{v}=-0 \%\end{array}$ & 1 & 10 & 5 & 0.5 & 178.7 & Line fault \\
\hline $\begin{array}{l}\text { Overcompensation } \\
\mathrm{v}=-10 \%\end{array}$ & 2.6 & 80 & $10 \mathrm{k}$ & 12.2 & 79.6 & Line fault \\
\hline $\begin{array}{l}\text { Overcompensation } \\
\mathrm{v}=-30 \%\end{array}$ & 2.6 & 10 & $100 \mathrm{k}$ & 305 & Excluding & Line fault \\
\hline
\end{tabular}

The compensation admittance angles of the unfaulted line are all in Quadrant I or Quadrant IV on the complex plane when the line fault is simulated. On the condition of no loss on the line or the ratio between the conductance and the susceptance is less than $1.5 \%$, compensation admittance will fall into the small circle. Also, the set admittance can be deliberately set as being different from the actual admittance on the line, for example, allowing the error to be above $1.5 \%$ for the purpose to simulate the measurement error. In this case, the compensation admittance angle will be strictly located on the imaginary axis, differing by at least $3.4^{\circ}$ from the boundary of the fault identification zone. This difference is enough to ensure the unfaulted line will not be wrongly identified as the faulted line. As for the faulted line, some typical fault conditions are summarized as shown in Table 1.

From the table, all the faulted lines can be accurately identified by combining the criteria of the compensation admittance ratio and the compensation admittance angle. A fault is identified as the busbar fault when all the line protections signal no fault alarm but the zero-sequence voltage still exists. The simulation also proves the capacity of the criterion to identify the busbar fault. Results show that, even if the worst happens, namely no loss in the line and the fixed value of zero-sequence susceptance error set above $1.5 \%$, the compensation admittance angle remains constantly located on the imaginary axis, instead of in the fault identification zone. If the judgment of each line is correct, busbar fault can be easily identified.

It should be noted that the setting of each criterion in this paper is not the only one; flexible adjustments are allowed according to the acceptable limit of the CT error and the active loss of the arc suppression coil.

In order to highlight the advantages of the proposed method, it is compared with the existing typical methods, namely the zero-sequence admittance method, zero-sequence active component method and the transient zero-sequence method. Concerning the case in the third line of Table 1, the calculating results of the zero-sequence admittance method, 
zero-sequence active component method and the transient zero-sequence method are expressed respectively in Table 2.

Table 2.The Performance of the Existing Approaches in Certain Situations

\begin{tabular}{|l|l|l|l|l|l|}
\hline \multirow{2}{*}{$\begin{array}{l}\text { Existing } \\
\text { Approach }\end{array}$} & $\begin{array}{l}\text { neutral grounded mode: overcompensation } \\
\mathrm{v}=40 \% \\
\text { damping ratio(\%): 0 } \\
\text { The ratio of the length of the faulted line to the } \\
\text { length of all lines }(\%) \text { : } 90 \\
\text { transition resistance }(\Omega): 100\end{array}$ & \begin{tabular}{c} 
Line \\
\cline { 2 - 5 }
\end{tabular} \\
\cline { 2 - 6 } & Line 1 & Line 2 & Line 3 & Line 4 & \\
\hline $\begin{array}{l}\text { Zero admittance } \\
\text { angle }\end{array}$ & 91.64 & 89.47 & 89.84 & 89.95 & 90.32 \\
\hline $\begin{array}{l}\text { Zero-sequence } \\
\text { active } \\
\text { component (kw) }\end{array}$ & -0.865 & 0.001 & 0.000 & -0.002 & 0.002 \\
\hline
\end{tabular}

As is indicated in Table 2, for the zero-sequence admittance method, it fails to function when all the admittance angles are near the imaginary axis in the admittance plane. Apart from that, with the admittance angle of the faulted line being $91.64^{\circ}$ and the maximum admittance angle of the unfaulted line being $90.32^{\circ}$, the difference between them amounts to above $90^{\circ}$ due to the measurement error. In this case, the faulted line cannot be accurately identified even if the action threshold is set as $90^{\circ}$ and without the consideration of the permissible error. For the zero-sequence active component method, theoretically, there exist the active power of the faulted line being negative and all the active power of the unfaulted line being positive. From Table 3-2, in addition to the fact that the active power of the faulted line is a negative maximum, there is a unfaulted line whose active power appears to be negative due to the quantization-error and stochastic-error. In this case, the method based on zero-sequence active power fails.

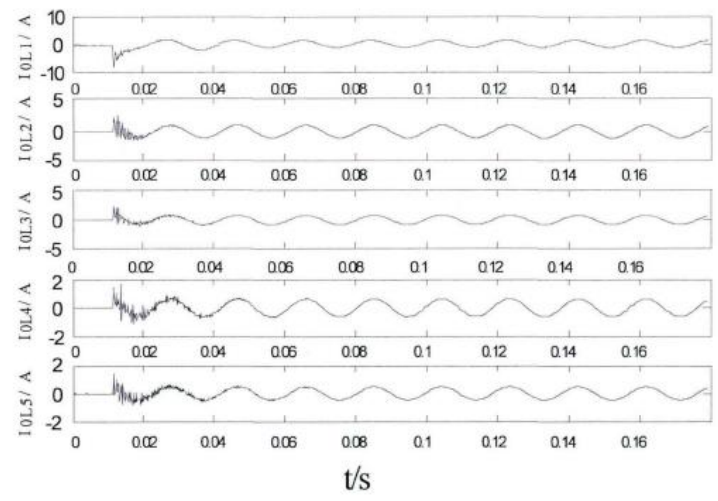

Figure 4. The Zero-sequence Current of the 5 Lines upon Single Phase Fault in Line 1

For the transient zero-sequence current method in this case, the oscillogram of the 5 lines is shown in Figure 4. 
From Figure 4, the transient maximum value of the faulted line is larger than the unfaulted line. In the meanwhile, the faulted line is opposite to other lines in terms of the direction of the transient maximum value. Therefore, the transient zero-sequence current method is effective in this case.

However, the method based on instantaneous sampling is often questioned by operators. Therefore, some anti-interference measures should be taken when this method is applied in practice.

\section{Conclusion}

Based on the study of the algorithm of zero-sequence, proposed in this paper are the concept of compensated admittance and the single phrase fault protection algorithm that combines the ratio of compensation admittance and the compensated admittance angle. This algorithm retains the advantage of the zero-sequence admittance method, which can identify the line fault independently. Meanwhile, the zero-sequence admittance method also overcomes the disadvantage of low sensitivity in the identification of the faulted line and the unfaulted line. Simulation tests prove that this algorithm can correctly distinguish the line fault, the busbar fault, and is not affected by the fault grounded resistance. This algorithm, not affected by the system structure and the pattern of neutral grounding, enables considerable improvements in the accuracy of the faulted line selection.

\section{References}

[1] D. Zhang, F. Wang and R. Burgos, "Impact of in-terleaving on AC passive components of paralleled three-phasevoltage-source converters", IEEE Transactions on Industry Applications, (2010).

[2] D. Zhang, F. Wang and R. Burgos, "DC-link rip-ple current reduction for paralleled three-phase voltage-sourceconverters with interleaving”, IEEE Transactions on Power E-lectronics, (2011).

[3] L. A. Serpa, S. Ponnaluri and P. M. Barbosa, "A Modified Direct Power Control Strategy Allowing the Connection of Three Phase Inverters to the Grid Through LCL Filters", IEEE Transactions on Industry Applications, (2007).

[4] I. J. Gabe, V. I. F. Montagner and H. Pinheiro, "Design and Implementation of a Roubst Current Con-troller for VSI Connected to the Grid Through an LCL filter”, IEEE Transactions on Power Electronics, (2009).

[5] F. R. Quintela, J. M. G. Arévalo, R. C. Redondo and N. R. Melchor, “ Four-wire three-phase load balancing with Static VAr Compensators [J]”, International Journal of Electrical Power and Energy Systems, vol. 3, (2011).

[6] JC Olivares-Galvan, PS Georgilakis and R Ocon-Valdez, "A review of transformer losses", Electr Power Comput Syst., (2009).

[7] KM Takami and J. Mahmoudi, "A novel study on the power transformer losses in relation to CO2capturing technology", (2008).

[8] N. Feng and Y. Jianming, "Low-Voltage Distribution Network Theoretical Line Loss Calculation System Based on Dynamic Unbalance in Three Phrases", Proceedings of the 2010 International Conference on Electrical and Control Engineering, (2010).

[9] B. Tomislav S`ekara and C`. Jovan Mikulovic', "Optimal Reactive Compensators in PowerSystems Under Asymmetrical and Nonsinusoidal Conditions", IEEE TRANSACTIONS ONPOWER DELIVERY, (2008). 
[10] X Y Cui, "Relation between the abnormal infrasound signals and the earthquakes [D]", Beijing: Beijing university of technology institute of mechanical and electrical, (2010).

[11] JF. Doyle, "Wave propagation in structure [M]", Springer, (1997).

[12] X. Gao, H. Luo and K. Hirahara, "Numerical modeling of stress generation and transfer caused by the Japanese subduction zone [J]”, Seismology and geology, vol. 16, no. 2, (1994), pp. 97-108.

[13] J. Xu, Z. Zhao and Y. Kono, "Regional characteristics of stress field and its dynamics in and around the Nankai trough, Japan [J]”, Chinese journal of geophysics, vol. 46, no. 4, (2003), pp. 488-494.

[14] K. Hirahara, "Interplate earthquake fault slip during periodic earthquake cycles in a viscoelastic medium at a subduction zone [J]”, Pure Appl. Geophys., vol. 159, (2002), pp. 2201-2220.

[15] S. Mei, B. Liang and Y. Zhu, "Digital simulation on precursory mechanism of the 1976 Tangshan earthquake [J]", Earthquake research in China, vol. 5, no. 2, (1989), pp. 27-34.

[16] Y. Geng, J. He and K. Pahlavan, "Modeling the Effect of Human Body on TOA Based Indoor Human Tracking[J]", International Journal of Wireless Information Networks, vol. 20, no. 4, (2013), pp. 306-317.

\section{Author}

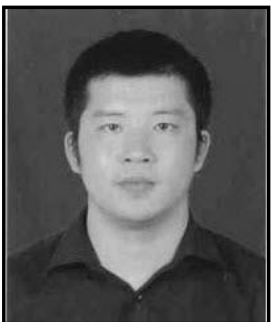

JiangJun Yuan, he was born in 1979, He is male, Shaoxing Zhejiang, Master, Associate Professor, the main research areas: e-commerce 